\title{
Review
}

\section{From PTK-STAT signaling to caspase expression and apoptosis induction}

\author{
$\mathrm{X}-\mathrm{Y} F \mathbf{u}^{*, 1}$ \\ 1 Department of Pathology, Yale University School of Medicine, New Haven, \\ Connecticut, CT 06520, USA \\ * Corresponding author: X-Y Fu, Department of Pathology, Yale University \\ School of Medicine, New Haven, Connecticut, CT 06520, USA, \\ Tel. 203-737-1246; Fax: 203-737-1247; E-mail: xin-yuan.fu@yale.edu
}

Received 13.5.99; revised 6.10.99; accepted 13.10.99 Edited by L Fesus

\begin{abstract}
The cell growth and survival can be regulated by cytokineinduced signals. Nevertheless, the molecular mechanisms of signal transduction from cytokine receptors to mediators of cell growth and apoptosis are not well characterized. STAT (signal transducer and activator of transcription) proteins can be activated by protein tyrosine kinases (PTK) in response to a variety of cytokines. We have shown that STAT activation induces expression of caspases which may cause cells undergo apoptosis. A hypothesis is proposed that PTK-STAT signaling pathways can regulate induction of apoptosis in response to cytokines and other extracellular signals.
\end{abstract}

Keywords: protein tyrosine kinase; STAT; apoptosis induction; caspase; gene regulation

Abbreviations: PTK, protein tyrosine kinase; STAT, signal transducer and activator of transcription; IL, interleukin; EGF, epidermal growth factor; JAK, Janus kinase; IFN, interferon; ICE, interleukin converting enzyme

\section{Introduction}

A living cell is constantly receiving and responding to a variety of extracellular signals. Important parts of these signals are polypeptides such as hormones, growth factors, cytokines, neural transmitters, antigens and cell matrix proteins. The state of cell metabolism, proliferation, differentiation, and other phenotypes is controlled by these polypeptide ligands. The signal transduction of these polypeptide ligands depends on their specific cell surface receptors. A ligand exerts its action by binding to its receptor on the cell surface and then initiating a chain of reactions within the cell through the intracellular domain of the receptor, which often contains certain enzymatic activities or/and associated with signaling proteins.

A living cell can also commit a programmed cell death or apoptosis in response to extracellular signals and internal defects, or during development and morphogenesis. ${ }^{1-4} \mathrm{~A}$ hallmark of the programmed cell death that differs from other types of cell death is the involvement of a specific set of cell death genes. Furthermore, it was known over 30 years ago that cell death during development and morphogenesis may require de novo gene expression. ${ }^{5}$ Therefore, a specific gene expression program may also be essential for induction of apoptosis.

It is well known that signals from cell surface receptors can regulate cell survival and death. A well known cell death signaling process is shown by studies of TNF receptor/FAS (CD95) related cell surface proteins. ${ }^{6,7}$ Activation of TNFR/Fas may induce a signaling cascade resulting in eventual activation of proteases, followed by proteolytic reactions and destruction of a cell. The key executors of apoptosis are the caspase family of cysteine proteases $^{8-10}$ which were recognized through cloning and functional analysis of the $C$. elegans programmed cell death gene ced-3. ${ }^{2,11}$

Apoptosis is also regulated by the $\mathrm{Bcl}-2$ family of genes. The Bcl-2 gene was first identified by cloning of the breakpoint of the chromosome 14-18 translocation, a characteristic of human follicular lymphoma. ${ }^{12}$ Some of these family members inhibit (Bcl-2 and Bcl-xL) apoptosis while the others (Bax, Bcl-xS, Bad) accelerate death. ${ }^{13}$ Abnormal regulation or mutations in members of $\mathrm{Bcl}-2$ family genes can affect cell survival and lead to tumorigenesis. How the Bcl-2 family of genes is regulated is not clearly understood.

Although tremendous progress has been made in recent years in identifying mediators of apoptosis, and in understanding molecular and biochemical steps in the execution of apoptosis through action of 'death genes,' very little is known about possible mechanisms involving regulation of the death genes, and what are the roles of the death gene expression in potentiation and induction of apoptosis. In particular, it has not been well studied whether there are signals and pathways that can control expression of death genes, and thus eventually determine the fate of a cell: death or survival.

Can the signals triggered by cytokines such as protein tyrosine kinase activation and gene expression be linked to the apoptosis? How are the expression of proteases and other 'death genes' regulated? Why are some kinds of cells more sensitive to induction of apoptosis than other types of cells? Can cytokines or other signals regulate expression of death genes? It is reasonable to suspect that the 'machine' for execution of apoptosis, such as caspase family proteins, can be regulated by specific transcription factors, which could be further modulated by cytokine-triggered signaling pathways. In other words, regulation of these death genes may provide a clue as to how the activation of specific signal transduction programs leading to death induction is 
achieved. The extent of death gene expression or repression might determine the fate of a cell: death or survival, or at least, the sensitivity of a cell to induction of apoptosis. Despite identification of many of the important players in the apoptosis process, there is much to learn about how these death genes are regulated.

Recently, it has been revealed that STAT (Signal Transducer and Activator of Iranscription ${ }^{14,15}$ can regulate gene expression of caspases and induce apoptosis in response to activation of protein tyrosine kinases (PTKs). ${ }^{16,17}$ These recent findings may provide a clue for the hidden mechanisms of potentiation and induction of apoptosis through regulation of caspase gene expression. Since the PTK-STAT pathway can be activated during cellular responses to polypeptide ligands, pathogens and other stimuli, it is tentative to postulate that regulation of apoptosis through this pathway may have a wide role in many pathological and physiological processes including degenerative diseases, and during development and morphogenesis.

In consideration of the readership of this article, I will first review briefly the history of discovering the PTK-STAT pathway, then I will discuss recent progress in understanding of cytokine and PTK-induced apoptosis. A hypothesis is proposed concerning the mechanism of PTK-induced apoptosis. I will also discuss the significance of this STAT-caspase connection in regulation of the programmed cell death.

\section{PTK activation induces diverse signaling pathways and cellular effects}

The central question is whether and how other cell surface receptors could regulate programmed cell death besides the 'death receptors.' Many growth factors and cytokines transduce signals through activation of protein tyrosine kinases (PTKs). Most growth factor receptors contain intrinsic tyrosine kinase domains, whereas cytokine receptors can be associated with cytoplasmic tyrosine kinases. ${ }^{18,19}$ Through some mediators or adapters, the tyrosine kinases can further activate a series of downstream serine/threonine kinases. These serine/threonine kinases in turn can stimulate effectors such as transcription factors in the cytoplasm and nucleus (reviewed in ${ }^{20,21}$ ).

A vast amount of evidence indicates that kinase cascade pathways play important roles in mediating cell proliferation, differentiation and survival. For example, activation of PTKs through ligand-mediated receptor dimerization can result in the activation of a number of downstream signaling pathways, including $\mathrm{PI} 3$ kinase pathway and Ras-Raf-MAP kinase pathways. The PI3 kinase and MAP kinase are believed to mediate cell survival and mitogenic responses. ${ }^{18,20,22}$ Most cytokines and growth factors can activate the PTK-Ras-MAP kinase and PTK-PI3 kinase pathways; thus, they can act as mitogens for a variety of cells.

Furthermore, deprivation of growth factors or cytokines can result in either cell growth arrest or apoptosis. Thus, growth factors, such as insulin-like growth factor-1 (IGF-1), EGF and PDGF are also regarded as survival factors. Many downstream signaling proteins, such as Ras, Raf, and nuclear transcription factors, such as Fos, Jun, Myc etc., have been shown to be oncogenes. ${ }^{21}$ Thus PTK signaling is generally believed to be involved in promoting proliferation and survival.

On the other hand, in contrast to the above concept that PTK signaling is involved in positive regulation of cell growth and survival, it is well documented that some PTKactivating growth factors and cytokines can induce apoptosis. Among them, interferon (IFN) can trigger cell cycle arrest and cell death. ${ }^{23}$ Furthermore, it is puzzling that EGF, which is an important growth factor, can also induce apoptosis in some mammalian cells. ${ }^{24,25}$ Additionally, many other cytokines such as IL-2 and IL-6, may also have the potential to induce apoptosis. ${ }^{26,27}$ Therefore, cytokines and PTK signal transduction may have dual, positive and negative, effects on cell growth and survival.

\section{Protein tyrosine kinase (PTK)-STAT pathway}

Studies on the mechanism of interferon-induced gene expression reveals a direct signaling pathway from cell surface receptors to the transcription factors (for review $\left.\operatorname{see}^{14,15,19}\right)$. In this pathway, the signal is mediated by STAT proteins which contain Src homology region $2(\mathrm{SH} 2)$ domains and can bind directly to the tyrosine phosphorylated receptors. ${ }^{28-31}$ STAT proteins are then phosphorylated and activated in the cytoplasm by PTKs, such as JAK tyrosine kinases. ${ }^{28,29,32-36}$ The activated transcription factors then translocate to the nucleus, joined by a nuclear DNA binding factor to form an active transcriptional complex. ${ }^{14,37,38}$

Although this signaling pathway was first revealed in the interferon system, it has been further demonstrated that most cytokines and growth factors, including EGF, PDGF, CSF-1, insulin, IL-2, IL-3, IL-4, IL-5, IL-6, IL-7, IL-9, IL-10, IL-11, IL-12, CSF, GM-CSF etc., can activate the direct STAT signaling pathway. ${ }^{19}, 29,35,39-47$ So far more than six members of the STAT protein family have been identified in higher eukaryotes, and these STAT proteins are believed to respond specifically to different cytokine and other extracellular signals. ${ }^{19,48}$

The Jak family tyrosine kinases were initially recognized as activators of STAT proteins. ${ }^{33-36}$ However, a variety of tyrosine kinases, such as EGF receptor tyrosine kinase and Src kinase have been shown to activate STAT proteins directly and independently of JAK kinases. ${ }^{29,49,50}$ Our recent work has further shown that FGF receptor tyrosine kinase, Lck kinase, focal adhesion kinase (FAK) and many other tyrosine kinases can also activate STAT proteins, ${ }^{51,52}$ (Xie and $\mathrm{Fu}$, unpublished results). It is possible that most protein tyrosine kinases have the potential to activate the STAT pathway under physiological conditions. Therefore, the PTK-STAT pathway may be a general route of signaling in response to a variety of polypeptide ligands and other stimuli.

\section{From PTK-STAT to induction of caspases and apoptosis}

After the initial discovery of the STAT signaling pathway, it is necessary to address several important questions about 
cellular functions of the STAT pathway. First, can activation of STAT proteins affect cell cycle control? It was first observed that some cell lines, such as A431 and MDA-MB-468, cells were growth inhibited by treatment of EGF. In these cell systems, EGF can activate STAT proteins at the same level as IFNs. Therefore, it was speculated that STAT activation by EGF and IFNs may cause cell growth arrest. In other words, the STAT pathway may have a negative role in regulation of cell growth. Experiments using STAT1-deficient cell lines have shown that STATs, in particular Stat1, can negatively regulate cell growth. ${ }^{16,53}$ Interestingly, it was shown that cell cyclin inhibitors, such as p21/WAF1, can be upregulated through the STAT pathway and result in eventual cell growth arrest. $^{16,54-57}$

Furthermore, it was noticed that the STAT activation may not only cause cell growth arrest, but also increase cell death in response to cytokine treatment. ${ }^{16}$ The initial observation we had was the correlation of apoptosis induction with the STAT activation in a number of cell lines that over express EGF receptor. Normally, in many types of cell lines, probably due to a specific inhibitor, EGF does not activate STATs. ${ }^{58}$ However, EGF receptor autophosphorylation and MAP kinase activity are all the same in all these 'conventional' cell lines. Interestingly, in some cancer cell lines that over-express the EGF receptor (such as A431 and MDA-MB-468 cells), that can probably overide the potential inhibitor for STAT activation, EGF activates the STAT pathway. Correlated with STAT activation, these cells undergo apoptosis after EGF treatment. $^{17}$

It was necessary to explore the cellular target(s) of the activated STAT for apoptosis induction. Among many genes we tested, ICE (Caspase-1) expression was most significantly upregulated in a STAT-dependent manner after either IFN- $\gamma$ or EGF treatment. Additionally a proteolytically cleaved form of ICE, p10, was also clearly observed in this cytokine induced apoptosis. The conclusive evidence for STAT-induced caspase and apoptosis was obtained using STAT-deficient cell lines. It was demonstrated that the Stat1 protein was defective in U3A cells. ${ }^{59,60}$ In these mutant cells, IFN- $\gamma$ treatment did not induce ICE gene expression. However, once a single gene of STAT1 was reintroduced into these cells, IFN- $\gamma$ restored its ability to induce ICE and apoptosis. Thus, we concluded that the STAT pathway is essential for the induction of caspase expression and apoptosis by IFN- $\gamma \cdot{ }^{17}$ Furthermore, these observations suggested a general model that the induction of caspase genes may be involved in cytokine-induced apoptosis. Our recent results have also indicated that several other caspases can also be upregulated through the STAT pathway (Asao and Fu, unpublished results). It is also reported that Stat1 may be required for constitutive expression of caspases, although the classical functions of Stat1 were believed not to be required. ${ }^{61}$ This basal level of caspases was necessary for induction of apoptosis by death signals such as TNF- $\alpha^{16}$ (Chin and Fu, unpublished observation). It was also shown that FAS and FAS ligand may also be upregulated by IFNs, possibly through Stat $1 .^{62,63}$ We have recently observed that activation of STAT5 can cause increased apoptosis in activated T cells. ${ }^{52}$ It is possible that STAT5 and other STAT proteins can also regulate genes involving apoptosis.

\section{Further unsolved questions}

Several important issues concerning the conclusions above should be further clarified here. First, we do not know at this point how the ICE gene or other caspase gene is regulated by the STAT proteins. In MDA-MB-468 cells, ICE mRNA was quickly induced (within $1 \mathrm{~h}$ ), indicating that STAT may directly regulate ICE gene expression. Since ICE gene induction could be much slower in other (such as A431) cells, an indirect regulatory mechanism might be involved. One possibility is through action of interferon regulatory factor-1, (IRF-1). ${ }^{64}$ It was shown that IRF-1 was one of the primary genes induced by cytokines through the STAT pathway. ${ }^{65,66}$ IRF-1 is believed required for ICE induction in response to radiation and DNA damage. ${ }^{67}$ We have identified several STAT binding site (SIE) or ISRE (interferon stimulated responsive element)-like sequences within $1 \mathrm{~Kb}$ of the $5^{\prime}$-flanking region of the ICE gene. However, the functional roles of these promoter elements are not fully characterized.

We have shown that apoptotic response in $\mathrm{ICE}^{-1-}$ cells was greatly reduced after IFN- $\gamma$ treatment. ${ }^{17}$ This observation may constitute evidence for the requirement for ICE in IFN- $\gamma$-induced cell death. Furthermore, although ICE is not essential for development as shown in $\mathrm{ICE}^{-1-}$ mice, ${ }^{68,69}$ it is involved in apoptosis resulting from several signals. For instance, ICE is involved in DNA damage-induced and IRF1-mediated apoptosis. ${ }^{69}$ Fas-induced apoptosis, ${ }^{68}$ granzyme B-induced apoptosis, ${ }^{70}$ degradation of basement membrane extracellular matrix (ECM)-induced apoptosis in mammary epithelial cells, ${ }^{71}$ and spontaneous cell death of thymocytes ( $\mathrm{T}$ Zheng and $\mathrm{R}$ Flavell, personal communication), and related in caspase 11 -mediated apoptosis. ${ }^{72}$ The convincing data were also obtained recently showing ICE is required for apoptosis of sensory neurons after growth factor withdrawal. ${ }^{73}$ It is very likely that elevated expression of ICE by cytokine treatment will lead to caspase activation and eventually cell death. However, while ICE expression is involved in the EGF- and IFN- $\gamma$ induced apoptosis as we have shown, the data do not imply that the up-regulation of the ICE gene alone is sufficient. Additionally, ICE is the first, but may not be the only caspase we have found to be regulated by the PTKSTAT signaling. Other members of caspases and other death genes may also be targets of regulation (Asao and Fu, unpublished data). For example, FAS and FAS ligands are also the possible targets genes of STAT proteins. ${ }^{63}$ Therefore, there must be multiple target genes in STATmediated apoptosis induction. Therefore the regulation of ICE by the STAT pathway can be regarded as the first evidence for a new hypothesis: the PTK-STAT pathway can regulate apoptosis.

Although in our initial systems we have first shown that Stat1 is involved in apoptosis induction in response to IFN and EGF, it by no means implies that Stat 1 is the sole member in the STAT family that has the potential for apoptosis induction. It is very likely that under different 
situations, other members of STATs may well play roles in apoptosis induction. Among other STAT proteins, STAT3 may well play a role in IL-6 induced apoptosis. ${ }^{74}$ Thus function of Stat1 could serve as a paradigm for the more general roles of other STATs. Moreover, although some caspases such as CPP32/caspase-3 may not be regulated by STAT proteins, ${ }^{17}$ its enzymatic activities may be activated by other caspases. The possible redundant functions of different members of STATs and caspases may provide an explanation to the observation that a single null mutation of one member of STATs or caspases may not cause significant defects in development. ${ }^{68,69,75,76}$

Since the PTK-STAT pathway plays essential roles in cellular responses to many pathological and physiological stimuli, it is possible that some diseases or developmental defects resulting from inappropriate cell arrest and apoptosis may be due to an overactive PTK-STAT pathway. Like JAK and EGF receptor kinases, focal adhesion kinase (FAK) and fibroblast growth factor (FGF) receptor tyrosine kinase can also activate Stat 1 and induce apoptosis. In particular, we have observed STAT activation by integrin-FAK signaling. Under the normal conditions, the STAT activation does not trigger apoptosis because it is rather weak and the surviving signals are strong in response to PTK activation. However, when survival signal was reduced by serum withdrawal, the negative effect induced STAT activation may promote apoptosis (see below).

\section{The molecular mechanisms of the dual activities of cytokines in cell growth and survival}

We have suggested that the STAT activation by protein tyrosine kinases can induce the expression of caspases and cause apoptosis. The model systems that were used are interferon and EGF responsive cells. However, do these findings have any general significance?

Many growth factors and cytokines have dual effects on cell growth and survival. ${ }^{77}$ IL-6 stimulates hepatocyte proliferation and prevents apoptosis; but IL- 6 inhibits cell growth and induces apoptosis in some myeloma cell lines, and in terminally differentiated B-cells. ${ }^{74,78}$ Like EGF, PDGF is required for proliferation and survival in many types of cells but also inhibits cell growth and accelerates apoptosis in some other cells. ${ }^{79}$

What is the molecular basis responsible for the dual functions of cytokines? A working model is that every PTKactivating cytokine simultaneously initiates multiple signaling pathways that may have opposite, positive and negative, effects on cell growth. Thus whether a cytokine promotes or inhibits cell growth is determined by the relative strengths of positive or negative signals that may be induced intrinsically by the same cytokine-bound receptor. In particular, activation of the Ras-MAP kinase and PI3-Akt kinase pathways promote cell growth/survival. In contrast, activation of the STAT pathway, especially STAT1 protein, may result in cell arrest/death by induction of CDK inhibitors and caspases. The intracellular homeostasis requires a balance between growth/survival and arrest/death signaling events. Different cells may have different dynamic states and hence different phenotypic outputs.

Take EGF as an example, EGF does not activate the STAT pathway in many types of cells, ${ }^{58}$ but EGF does trigger the MAP kinase pathway etc., in these cells. Therefore, the positive signals are dominant, and these cells proliferate and survive in response to EGF. However, in A431 or MDA-MB-468 cells, the STAT pathway is more sensitive in response to EGF, so CDK inhibitors and the caspase can be highly induced, which may override the positive signals and result in cell arrest and death. Therefore, EGF can conditionally activate a negative signaling pathway through STAT activation and expression of CDK inhibitors and caspases in cells like A431 and MDA-MB-468.

Thus, the polypeptide ligand-activated PTK signaling could not only transduce the proliferative and surviving signals, as most commonly observed, but also, under some conditions, generate anti-proliferative and cell death signals that are mediated by the STAT proteins, STAT1 in particular. This conclusion may provide a possible explanation for the dual functions of many cytokines and growth factors.

Interestingly, TNF- $\alpha$ signaling was also found to initiate at least two opposing pathways: induction of apoptosis through the activation of a protease cascade and activation of transcription factor NF- $\kappa \mathrm{B}$ which suppresses apoptosis. ${ }^{80-83}$ However, unlike TNF- $\alpha$-induced apoptosis in which gene expression may not be directly required, PTKSTAT-induced apoptosis processes require coordinate expression of specific gene(s), such as caspases.

\section{Puzzles and questions of apoptosis induction after serum withdrawal}

The new concept that PTK signaling itself can lead to apoptosis, may have further implications for the unsolved puzzle that cell death can be triggered by growth factor deprivation, and 'spontaneous' apoptosis during development and morphogenesis and some human degenerative diseases.

It is important to determine the mechanisms of cell death when the necessary cytokines, growth factors, or cell matrix proteins are not provided. This induction of apoptosis after growth factor withdrawal has been believed to be due to a 'default' mechanism. ${ }^{84}$ According to this notion, cells can only survive when growth factors are provided to suppress this mechanism to die. What is the molecular basis of this default mechanism? Can this 'default' be regulated and affected by signaling pathways? What are the mediators that carry out this default mechanism? None of these questions have answers yet.

The cell death induced by growth factor deprivation may also involved in development. Lack of growth factors or other stimuli is believed to be responsible for organogenesis and maintenance of homeostasis of the whole body. Furthermore, many kinds of degenerative diseases are believed to be caused by 'spontaneous' apoptosis, an event resembling cell death triggered by growth factor withdrawal. $^{3}$ 
A critical question is whether the molecular mechanism of the apoptosis induced by cytokine treatment can also mediate the cell death or apoptosis triggered by cytokine deprivation. What are the hidden molecular mechanisms controlling the induction of apoptosis in either the presence or the absence of cytokines and growth factors? Could these two seemingly opposite observations, death induced by cytokine deprivation or death through treatment of cytokines in some cells, have a common molecular basis?

\section{A hypothesis on apoptosis induced by growth factor withdrawal}

As I mentioned in the previous sections, many studies of signal transduction to apoptosis have focused on TNF/FAS mediated apoptosis that may not require de novo gene expression. However, more and more cases have been documented in which the induction of apoptosis under many conditions may involve distinctive signals other than those elicited by TNF/ FAS. One of most striking examples is induction of apoptosis after the deprivation of growth factor or other survival factors. Their signals are apparently different from those by TNF/FAS: it is a slower process, and the up or down regulation of certain genes is possible. However, little was known about the mechanisms and possible signaling process. Although the concept of apoptosis by default is an attractive model, it did not provide the molecular basis for the 'default.' For example, what are the specific signals and executors that may be responsible for the intrinsically hard-wired machine for induction of apoptosis? In other words, there must be certain mediators or gene products to carry out the death execution after survival factor deprivation, based on the view that apoptosis is an active process. What are these potential genes? Most interestingly, can expression of these death genes be regulated by certain signaling pathways? It is known that for different types of cells there are significant differences in their sensitivities or thresholds for apoptosis induction by default. ${ }^{3}$ Then how are these thresholds for apoptosis induction determined? These are significant and fundamental questions. If we can find clues for the apoptosis induction by this default mechanism after survival signal withdrawal, it may provide mechanisms for many important situations of apoptosis induction during stages of development or in the pathogenesis of auto immune disorders, leukemia, and some degenerative diseases, and for many other types of 'spontaneous' apoptosis.

Here, I propose a hypothesis that the PTK-STAT signaling pathway may be an essential player in induction of apoptosis by default after the withdrawal of the survival signal. As I have shown above, the PTK-STAT pathway can mediate induction of apoptosis through the regulation of caspases and other cell death genes. Under the conditions we used, the PTK-STAT can be strongly activated and the induction of caspases is high. Therefore, the survival signals from PTK are overcome by the effects of STAT activation and upregulated caspases, causing apoptosis. However, in normal cell culture conditions without a large amount of cytokines, STAT proteins may only

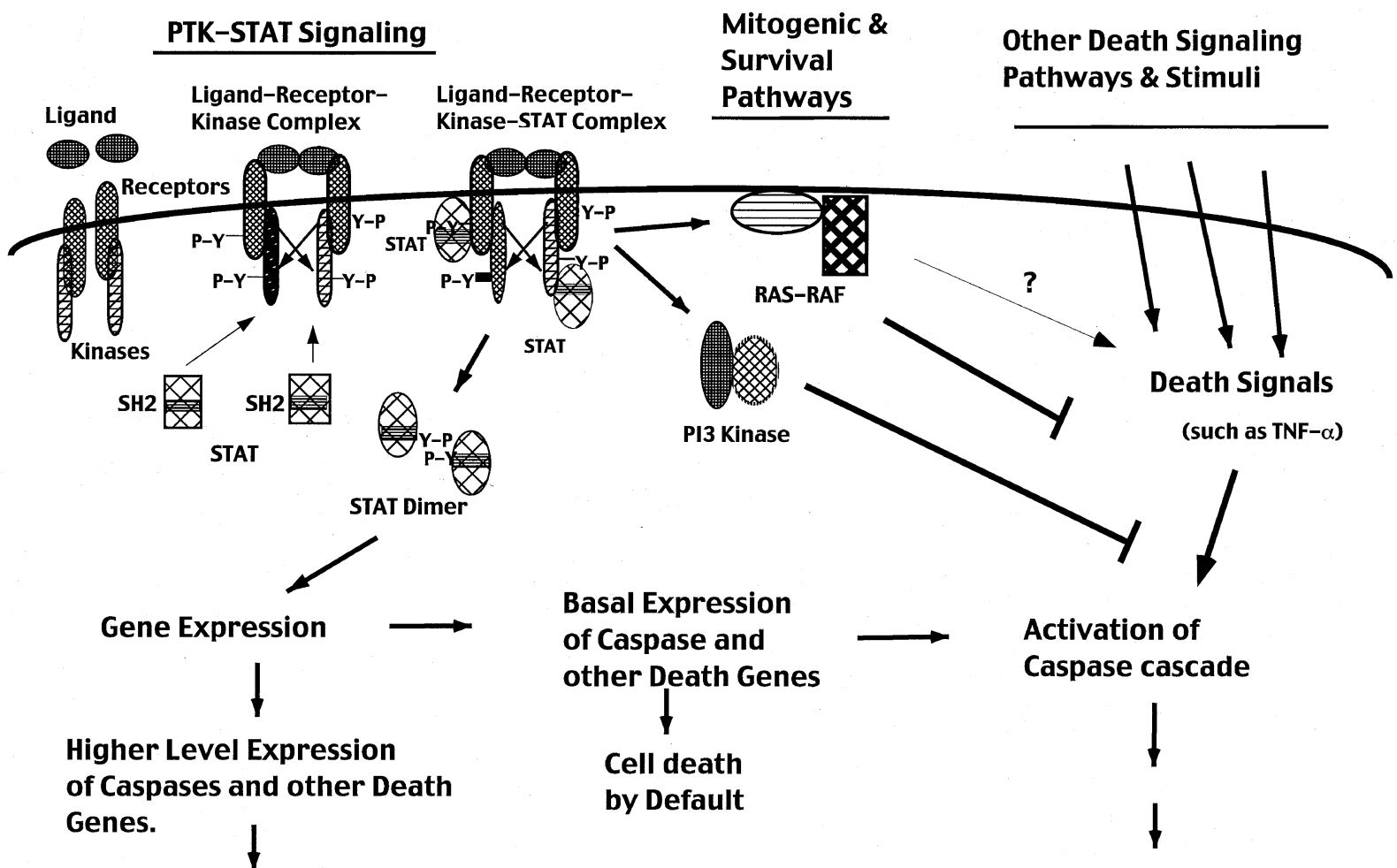

Committed to Die
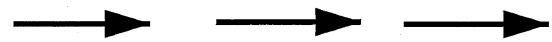

Execution of Cell Death

Figure 1 Signaling pathways to induction of apoptosis 
be activated at a lower level (possibly due to growth factors in the serum supplied, or matrix/integrin interactions etc. (Xie and $\mathrm{Fu}$ unpublished data). Coordinately, a lower and constitutive level of caspases is maintained which is not strong enough to overcome the survival signals. Thus these cells survive. However, when the survival signals are deprived by growth factor withdrawal, the balance between death signals (STAT and caspase activities) and survival signals may shift towards death. For example, MAPK activation may only last for 15-30 min, whereas STAT activation may last for hours (Asao and $\mathrm{Fu}$, unpublished data). Thus the differences between positive and negative signals after growth factor withdrawal can be a decisive factor for the balance shifting to death. In this model, the hard-wired, intrinsic executioner or mediator (THE DEFAULT) of apoptosis is the low level of STAT and caspase activities regulated by STAT. Furthermore, the differences in the thresholds for induction of apoptosis may be determined by the different levels of STAT and caspase activities. Once the survival signals for suppressing these death mediators are weakened after growth factor withdrawal, it is the turn for action of STAT and caspase and other death genes, and the apoptosis occurs.

\section{A brief summary and perspectives}

We are at the beginning of understanding of the STAT functions in control of apoptosis. The hypothesis proposed above is only one possible example. I summarize some thoughts on possible STAT functions in apoptosis shown in Figure 1 and give some perspectives as follows:

(1) Cytokines, such as IFNs that activate the PTK-STAT pathway, can cause a higher level of expression of caspases and other death genes. When the signal is strong enough (for example, high level expression of caspases may trigger autocleavage and activation of caspase cascade), the PTK-STAT pathway itself will be a major driving force for induction of apoptosis.

(2) Under the basal conditions, STAT proteins are activated at a low level. For instance, in the cultured cells, the growth factors in the serum-supplemented media may be responsible for so-called 'constitutive' or basal activities of STAT proteins. Accordingly, a 'constitutive' level of expression of caspase and other death genes is maintained through the basal activity of STAT proteins in these cells. These constitutively expressed caspases are necessary and required for the activation of the caspase cascade, leading to execution of apoptosis in response to a variety of death signals and stimuli, such as TNF family of cytokines. As discussed above, this basal level of STAT and resultant caspase activities may be also essential for apoptosis induction by default.

(3) Under different cell conditions or context, and dependent on the signal strengths, STAT activation may make cells more committed for induction of apoptosis and increase the sensitivity of the cells to other apoptotic signals. However, additional death signals are further required for triggering apoptosis. Thus, STATs may play a more general role in apoptosis induction during a variety of physiological and pathological processes. It is anticipated that defective in the STAT function may cause insufficient apoptosis, whereas increased and lasting STAT activities may be a critical factor responsible for enhanced apoptosis. Since several members of STAT proteins (STAT1, STAT3 and STAT5 which share similar DNA binding sites) may be involved in control of apoptosis genes, knock-out one of STAT genes may not be sufficient to show the phenotype of apoptosis defect and the double or triple knock-outs need to be performed and examined. However, in specific tissues or cells, one member of STAT proteins may play a major role (such as STAT5 in the $T$ cell activation), ${ }^{52}$ the defect in apoptosis induction should be still observable. I expect that more thorough analysis of STAT-deficient mice during the development and under relevant physiological and pathological conditions will reveal more evidence on the critical role of STAT proteins in apoptosis induction. As transcription factors, STAT proteins may regulate directly genes that are involved in induction of apoptosis in response to the PTK activation. However, it is also likely that STAT proteins may control and activate other transcription factors, such as IRF-1, that will further regulate apoptosis genes such as caspases.

(4) The PTK activation by polypeptide ligands will activate mitogenic and survival pathways in parallel of activation of STAT proteins. These positive pathways (such as RAS-RAF, and PI3 kinase) have suppressive effects on induction of apoptosis (RAS activation may also facilitate apoptosis under some conditions). The balance between the negative signals and the positive signals will determine the statues of the cell, apoptosis or survival. The default apoptosis may not occur through action of STATs and caspases until the balance is broke as a result of faster loss of survival signals than death signals.

(5) Finally, although our data indicated that the activation of STAT proteins may cause expression of death genes, resulting in higher sensitivity to apoptosis induction, it is possible that STAT proteins may also regulate genes that suppress induction of apoptosis. It is reported that $\mathrm{Bcl}-\mathrm{xL}$, a gene involved in cell survival, may be an immediate gene regulated by STAT proteins, ${ }^{85,86}$ (Welte and $\mathrm{Fu}$, unpublished observations). Furthermore, cyclin $D$ family members were shown to be directly regulated by STAT. ${ }^{87}$ In contrast to these early genes which are transiently induced by STAT, caspase gene induction through STAT is often a late and lasting process (mainly ranges from 1 to $48 \mathrm{~h}$ depending on cell types) ${ }^{17}$ (Asao and Fu, unpublished results). Thus, when a cytokine or other ligand activates PTKs, initial functions of PTK-STAT activities may facilitate cell survival and proliferation. If the stimulation continues and is further intensified, the later genes, including death genes, are expressed, which may overcome the effects of the early genes, resulting in termination of initial functions, and often, induction of apoptosis. This is a possible timely and efficient self-controlled 
mechanism of cells: The same signal, the PTK-STAT activation in response to cytokines and other polypeptide ligands, is contributing to both activation and destruction of the cell. Furthermore, the dual functions of the PTK-STAT pathway in regulating both survival/ proliferative and death genes may also provide a molecular mechanism for the widely-observed dual effects of cytokines and other polypeptide ligands for promoting cell proliferation/survival or for cell death.

\section{References}

1. Wyllie AH, Ker JFR and Currie AR (1980) Cell death: The significance of apoptosis. Int. Rev. Cytol. 68: 251-270

2. Ellis RE, Yuan J and Horvitz HR (1991) Mechanisms and functions of cell death. Annu. Rev. Cell Biol. 7: 663-698

3. Thompson CB (1995). Apoptosis in the pathogenesis and treatment of disease. Science 267: $1456-1462$

4. Vaux DL and Korsmeyer SJ (1999) Cell Death in Development. Cell 96: 245-254

5. Tata JR (1966). Requirement for RNA and protein synthesis for induced regression of the tadpole tail in organ culture. Dev. Biol. 13: 77-94

6. Nagata $S$ and Golstein $P$ (1995) The Fas death factor. Science 267: 1449- 1456

7. Ashkenazi A and Dixit VM (1998) Death receptors: signaling and modulation. Science 281: $1305-1308$

8. Alnemri ES, Livingston DJ, Nicholson DW, Salvesen G, Thornberry NA, Wong WW and Yuan J (1996) Human ICE/Ced-3 protease nomenclature. Cell 87: 171

9. Salvesen GS and Dixit VM (1997). Caspases: intracellular signaling by proteolysis. Cell $91: 443-446$

10. Thornberry NA and Lazebnik Y (1998) Caspases: enemies within. Science 281: $1312-1316$

11. Yuan J, Shaham S, Ledoux S, Ellis HM and Horvitz HR (1993) The C. elegans cell death gene ced-3 encodes a protein similar to mammalian interleukin- 1 betaconverting enzyme. Cell 75: 641-652

12. Korsmeyer SJ (1995) Regulator of cell death. Trends in Genetics 11: 401-405

13. Reed JC (1995) Regulation of apoptosis by bcl-2 family proteins and its role in cancer and chemoresistance. Curr. Opin. Oncol. 7: 541-546

14. Darnell Jr JE, Kerr IM and Stark GR (1994) Jak-STAT pathways and transcriptional activation in response to IFNs and other extracellular signaling proteins. Science 264: $1415-1421$

15. Fu X-Y (1995) A direct signaling pathway through tyrosine kinase activation of SH2 domain-containing transcription factors. J. Leukocyte Biol. 57: 529-535

16. Chin YE, Kitagawa M, Su W-CS, You Z-H, Iwamoto Y and Fu X-Y (1996) Cell growth arrest and induction of cyclin-dependent kinase inhibitor p21WAF1/CIP1 mediated by STAT1. Science 272: 719-722

17. Chin YE, Kitagawa M, Kuida K, Flavell RA and Fu XY (1997) Activation of the STAT signaling pathway can cause expression of caspase 1 and apoptosis. Mol. Cell. Biol. 17: 5328-5337

18. van der Geer P, Hunter T and Lindberg R (1994) Receptor protein tyrosine kinases and their signal transduction pathways. Annu. Rev. Cell. Biol. 10: 251 337

19. Leonard WJ and O'Shea JJ (1998) Jaks and STATs: biological implications. Annu. Rev. Immunol. 16: 293-322

20. Schlessinger J and Ullrich A (1992) Growth factor signaling by receptor tyrosine kinases. Neuron 9: 383-391

21. Karin M and Hunter T (1995) Transcriptional control by protein phosphorylation: signal transmission from the cell surface to the nucleus. Curr. Biol. 5: 747-757

22. Franke TF, Kaplan DR and Cantley LC (1997) PI3K: downstream AKTion blocks apoptosis. Cell 88: $435-437$

23. Kimchi A (1992) Cytokine triggered molecular pathways that control cell cycle arrest. J. Cell. Biochem. 50: 1-9

24. Buick RN, Filmus J and Church JG (1991) The role of epidermal growth factor receptors in breast cancer. Cancer Treatment \& Research 53: 159-170

25. Brabyn CJ and Kleine LP (1995) EGF causes hyperproliferation and apoptosis in T51B cells: involvement of high and low affinity EGFR binding sites. Cell Signal 7: $139-150$

26. Lenardo MJ (1991) Interleukin-2 programs mouse alpha beta T lymphocytes for apoptosis. Nature 353: 858-861
27. Hirano T, Nakajima K and Hibi M (1997) Signaling mechanisms through gp130: a model of the cytokine system. Cytokine Growth Factor Rev. 8: $241-252$

28. Fu X-Y (1992) A transcription factor with SH2 and SH3 domains is directly activated by an interferon $\alpha$-induced cytoplasmic protein tyrosine kinase(s). Cell 70: $323-335.47$

29. FuX-Y and Zhang J-J (1993) Transcription factorp91 interacts with the epidermal growth factor receptor and mediates activation of the $c$-fos gene promoter. Cell 74: $1135-1145$

30. Greenlund AC, Farrar MA, Viviano BL and Schreiber RD (1994) Ligand-induced IFN gamma receptor tyrosine phosphorylation couples the receptor to its signal transduction system (p91). EMBO J. 13: 1591-1600

31. StahI N, Farruggella TJ, Boulton TG, Zhong Z, Darnell JrJE and Yancopoulos GD (1995) Choice of STATs and other substrates specified by modular tyrosinebased motifs in cytokine receptors. Science 267: 1349-1353

32. Schindler C, ShuaiK, Prezioso VR and Darnell JrJE (1992) Interferon-dependent tyrosine phosphorylation of a latent cytoplasmic transcription factor. Science 257: 809-813

33. Velazquez L, Fellous M, Stark GR and Pellegrini S (1992) A protein tyrosine kinase in the interferon $\alpha / \beta$ signaling pathway. Cell 70: 313-322

34. Muller M, Briscoe J, Laxton C, Guschin D, Ziemiecki A, Silvennoinen O, Harpur AG, Barbieri G, Witthuhn BA, Schindler C, Pellegrini S, Wilks AF, Ihle JN, Stark GR and Kerr IM (1993) The protein tyrosine kinase JAK1 complements defects in interferon-a/b and $-\mathrm{g}$ signal transduction. Nature 366: 129-135

35. Ihle JN, Witthuhn BA, Quelle FW, Yamamoto K and Silvennoinen O (1995) Signaling through the hematopoietic cytokine receptors. Annu. Rev. Immunol. 13: $369-398$

36. Ihle JN (1995) Cytokine receptor signalling. Nature 377: 591-594

37. Fu X-Y, Kessler DS, Veals SA, Levy DF and Darnell Jr JE (1990) ISGF3, the transcriptional activator induced by interferon $a$, consists of multiple interacting polypeptide chains. Proc. Natl. Acad Sci. USA 87: 8555-8559

38. Kessler DS, Veals SA, Fu XY and Levy DE (1990) Interferon-a regulates nuclear translocation and DNA-binding affinity of ISGF-3; a multimeric transcriptional activator. Genes Dev. 4: 1753-1765

39. Sadowski HB, Shuai K, Darnell Jr JE and Gilman MZ (1993) A common nuclear signal transduction pathway activated by growth factor and cytokine receptors. Science 261: $1739-1744$

40. Silvennoinen O, Ihle JN, Schlessinger J and Levy DE (1993) Interferon-induced nuclear signalling by Jak protein tyrosine kinases. Nature 366: 583-585

41. Gaffen SL, Lai SY, Xu W, Gouilleux F, Groner B, Goldsmith MA and Greene WC (1995) Signaling through the interleukin 2 receptor beta chain activates a STAT5-like DNA-binding activity. Proc. Natl. Acad. Sci. USA 92: 7192-7196

42. Hou J, Schindler U, Henzel WJ, Wong SC and McKnight SL (1995) Identification and purification of human Stat proteins activated in response to interleukin-2. Immunity 2: 321-329

43. Jacobson NG, Szabo SJ, Weber-NordtRM, Zhong Z, Schreiber RD, Darnell JrJE and Murphy KM (1995) Interleukin 12 signaling in T helper type 1 (Th1) cells involves tyrosine phosphorylation of signal transducer and activator of transcription (Stat)3 and Stat4. J. Exp. Med. 181: 1755-1762

44. Larner AC, David M, Feldman GM, Igarashi K, Hackett RH, Webb DSA, Sweitzer SM, Petricoin EFd and Finbloom DS (1993) Tyrosine phosphorylation of DNA binding proteins by multiple cytokines. Science 261: 1730-1733

45. Quelle FW, Shimoda K, Thierfelder W, Fischer C, Kim A, Ruben SM, Cleveland JL, Pierce JH, Keegan AD, Nelms K, et al. (1995a) Cloning of murine Stat6 and human Stat6, Stat proteins that are tyrosine phosphorylated in responses to IL-4 and IL-3 but are not required for mitogenesis. Mol. Cell. Biol. 15: 3336-3343

46. Ruff-Jamison S, Chen Kand Cohen S (1995) Epidermal growth factor induces the tyrosine phosphorylation and nuclear translocation of Stat 5 in mouse liver. Proc. Natl. Acad. Sci. USA 92: 4215-4218

47. Frank DA, Robertson MJ, Bonni A, Ritz J and Greenberg ME (1995) Interleukin 2 signaling involves the phosphorylation of Stat proteins. Proc. Natl. Acad. Sci. USA 92: $7779-7783$

48. Darnell Jr JE (1997) STATs and gene regulation. Science 277: 1630-1635

49. Quelle FW, Thierfelder W, Witthuhn BA, Tang B, Cohen S and Ihle JN (1995b) Phosphorylation and activation of the DNA binding activity of purified Stat 1 by the Janus protein-tyrosine kinases and the epidermal growth factor receptor. J. Biol. Chem. 270: 20775-20780

50. Yu CL, Meyer DJ, Campbell GS, Larner AC, Carter-Su C, Schwartz J and Jove R (1995) Enhanced DNA-binding activity of a Stat3-related protein in cells transformed by the Src oncoprotein. Science 269: 81-83 
51. Su WC, Kitagawa M, Xue N, Xie B, Garofalo S, Cho J, Deng C, Horton WA and Fu XY (1997) Activation of Stat1 by mutant fibroblast growth-factor receptor in thanatophoric dysplasia type II dwarfism. Nature 386: 288-292

52. Welte T, Leitenberg D, Dittel BN, al-Ramadi BK, BX, Hansen WR, Janeway J, C A, Bothwell ALM, Bottomly K and Fu X-Y (1999) PTK-STAT signaling pathway plays essential roles in T cell activation in response to antigen stimulation. Cold Spring Harbor Symposia on Quantitative Biology 64: In press

53. Bromberg JF, Horvath CM, Wen Z, Schreiber RD and Darnell Jr JE (1996) Transcriptionally active Stat 1 is required for the antiproliferative effects of both interferon alpha and interferon gamma. Proc. Natl. Acad. Sci. USA 93: 76737678

54. Matsumura I, Ishikawa J, Nakajima K, Oritani K, Tomiyama Y, Miyagawa J, Kato T, Miyazaki H, Matsuzawa Y and Kanakura Y (1997) Thrombopoietin-induced differentiation of a human megakaryoblastic leukemia cell line, CMK, involves transcriptional activation of p21(WAF1/Cip1) by STAT5. Mol. Cell. Biol. 17 2933-2943

55. Xie W, Su K, Wang D, Paterson AJ and Kudlow JE (1997) MDA468 growth inhibition by EGF is associated with the induction of the cyclin-dependent kinase inhibitor p21WAF1. Anticancer Res. 17: 2627-2633

56. Bellido T, O'Brien CA, Roberson PK and Manolagas SC (1998) Transcriptiona activation of the 21 (WAF1,CIP1,SDI1) gene by interleukin- 6 type cytokines. A prerequisite for their pro- differentiating and anti-apoptotic effects on human osteoblastic cells. J. Biol. Chem. 273: 21137-21144

57. Boccaccio C, Ando M, Tamagnone L, Bardelli A, Michieli P, Battistini C and Comoglio PM (1998) Induction of epithelial tubules by growth factor HGF depends on the STAT pathway. Nature 391: 285-288

58. Iwamoto $Y$, Chin YE, Peng $X$ and Fu XY (1998) Identification of a membraneassociated inhibitor(s) of epidermal growth factor-induced signal transducer and activator of transcription activation. J. Biol. Chem. 273: 18198-18204

59. McKendry R, John J, Flavell D, Muller M, Kerr IM and Stark GR (1991) Highfrequency mutagenesis of human cells and characterization of a mutant unresponsive to both alpha and gamma interferons. Proc. Natl. Acad. Sci. USA 88: $11455-11459$

60. Leaman DW, Leung S, Li X and Stark GR (1996) Regulation of STAT-dependent pathways by growth factors and cytokines. FASEB J. 10: 1578-1588

61. Kumar A, Commane M, Flickinger TW, Horvath CM and Stark GR (1997) Defective TNF-a-induced apoptosis in STAT1-null cells due to low constitutive levels of caspases. Science 278: 1630-1632

62. Der SD, Zhou A, Williams BRG and Silverman RH (1998) Identification of genes differentially regulated by interferon alpha, beta, or gamma using oligonucleotide arrays [In Process Citation]. Proc. Natl. Acad. Sci. USA 95: 15623-15628

63. Xu X, Fu XY, Plate J and Chong AS (1998) IFN-gamma induces cell growth inhibition by Fas-mediated apoptosis: requirement of STAT1 protein for upregulation of Fas and FasL expression. Cancer Res. 58: 2832-2837

64. Taniguchi T, Harada $\mathrm{H}$ and Lamphier M (1995) Regulation of the interferon system and cell growth by the IRF transcription factors. J. Cancer Res. Clin. Oncol. 121: $516-520$

65. Fujita T, Reis LFL, Watanabe N, Kimura Y, Taniguchi T and Vilcek J (1989) Induction of the transcription factor IRF-1 and interferon-b mRNAs by cytokines and activators of second-messenger pathways. Proc. Natl. Acad. Sci. USA 86: 9936- 9940

66. Pine R, Canova A and SchindlerC (1994) Tyrosine phosphorylated p91 binds to a single element in the ISGF2/IRF-1 promoter to mediate induction by IFN alpha and IFN gamma, and is likely to autoregulate the p91 gene. EMBO J. 13: 158167

67. Tamura T, Ishihara M, Lamphier MS, Tanaka N, Oishi I, Aizawa S, Matsuyama T, Mak TW, Taki S and Taniguchi T (1995) An IRF-1-dependent pathway of DNA damage-induced apoptosis in mitogen-activated T lymphocytes. Nature 376 : 596-599

68. Kuida K, Lippke JA, Ku G, Harding MW, Livingston DJ, Su MS and Flavell RA (1995) Altered cytokine export and apoptosis in mice deficient in interleukin-1 beta converting enzyme. Science 267: 2000-2003
69. Li P, Allen H, Banerjee S, Franklin S, Herzog L, Johnston CX, McDowell J, Paskind M, Rodman L, Salfeld J, Towne E, Tracey D, Wardwell S, Wei FY, Wong W, Kamen R and Seshadri T (1995) Mice deficient in IL-1 beta-converting enzyme are defective in production of mature IL-1 beta and resistant to endotoxic shock. Cell 80: 401-411

70. Shi L, Chen G, MacDonald G, Bergeron L, Li H, Miura M, Rotello RJ, Miller DK, Li P, Seshadri T, Yuan J and Greenberg AH (1996) Activation of an interleukin 1 converting enzyme-dependent apoptosis pathway by granzyme B. Proc. Natl. Acad. Sci. USA 93: 11002-11007

71. Boudreau N, Sympson CJ, Werb Z and Bissell MJ (1995) Suppression of ICE and apoptosis in mammary epithelial cells by extracellular matrix. Science 267:891 893

72. Wang S, Miura M, Jung YK, Zhu H, LiE and Yuan J (1998) Murine caspase-11, an ICE-interacting protease, is essential for the activation of ICE. Cell 92: 501-509

73. Friedlander RM, Brown RH, Gagliardini V, Wang J and Yuan J (1997) Inhibition of ICE slows ALS in mice. Nature 388: 31

74. Minami M, Inoue M, Wei S, Takeda K, Matsumoto M, Kishimoto T and Akira S (1996) STAT3 activation is a critical step in gp130-mediated terminal differentiation and growth arrest of a myeloid cell line. Proc. Natl. Acad. Sci. USA 93: 3963-3966

75. Durbin JE, Hackenmiller R, Simon MC and Levy DE (1996) Targeted disruption of the mouse Stat 1 gene results in compromised innate immunity to viral disease. Cell 84: 443-450

76. Meraz MA, White JM, Sheehan KC, Bach EA, Rodig SJ, Dighe AS, Kaplan DH, Riley JK, Greenlund AC, Campbell D, Carver-Moore K, DuBois RN, Clark R, Aguet $M$ and Schreiber RD (1996) Targeted disruption of the Stat1 gene in mice reveals unexpected physiologic specificity in the JAK-STAT signaling pathway. Cell 84: $431-442$

77. Sporn MB and Roberts $A B$ (1988) Peptide growth factors are multifunctional. Nature 332: 217-219

78. Altmeyer A, Simmons RC, Krajewski S, Reed JC, Bornkamm GW and ChenKiang S (1997) Reversal of EBV immortalization precedes apoptosis in IL-6induced human $B$ cell terminal differentiation. Immunity 7:667-677

79. Kim HR, Upadhyay S, Li G, Palmer KC and Deuel TF (1995) Platelet-derived growth factor induces apoptosis in growth-arrested murine fibroblasts. Proc. Natl. Acad. Sci. USA 92: 9500-9504

80. Beg AA and Baltimore D (1996) An essential role for NF- $\kappa$ B in preventing TNFinduced cell death. Science 274: $782-784$

81. Liu Z-G, Hsu H, Goeddel DV and Karin M (1996) Dissection of TNF receptor 1 effector functions: JNK activation is not linked to apoptosis, while NF- $\kappa$ B activation prevents cell death. Cell 87: 565-575

82. Van Antwerp DJ, Martin SJ, Kafri T, Green DR and Verma IM (1996) Suppression of TNF-alpha-induced apoptosis by NF-kappaB. Science 274: 787-789

83. Wang CY, Mayo MW and Baldwin ASJ (1996) TNF- and cancer therapy-induced apoptosis: potentiation by inhibition of NF-kappaB. Science 274: 784-787

84. Raff MC (1992) Social controls on cell survival and cell death. Science 356: $397-$ 400

85. Catlett-Falcone R, Landowski TH, Oshiro MM, Turkson J, Levitzki A, Savino R, Ciliberto G, Moscinski L, Fernandez-Luna JL, Nunez G, Dalton WS and Jove R (1999) Constitutive activation of Stat3 signaling confers resistance to apoptosis in human U266 myeloma cells. Immunity 10: 105-115

86. Dumon S, Santos SC, Debierre-Grockiego F, Gouilleux-Gruart V, Cocault L, Boucheron C, Mollat P, Gisselbrecht S and Gouilleux F (1999) IL-3 dependent regulation of $\mathrm{Bcl}-\mathrm{xL}$ gene expression by STAT5 in a bone marrow derived cell line. Oncogene 18: 4191-4199

87. Matsumura I, Kitamura T, Wakao H, Tanaka H, Hashimoto K, Albanese C, Downward J, Pestell RG and Kanakura Y (1999) Transcriptional regulation of the cyclin D1 promoter by STAT5: its involvement in cytokine-dependent growth of hematopoietic cells. EMBO J. 18: 1367-1377 